\title{
Ultrafiltration Technology and Its Application in
}

\section{Cardiopulmonary Bypass}

\author{
Kun Zhang, Lishen Wang* and Shuwei Shen \\ Department of Anesthesiology, Jingzhou Central Hospital, The Second Clinical Medical College, Yangtze University, \\ Jingzhou, Hubei Province, 434020, China \\ Corresponding E-mail: 7885909@qq.com
}

\begin{abstract}
The application of cardiopulmonary bypass (CPB) technology makes it possible to safely conduct open-heart surgery. However, as an invasive adjuvant therapeutic measure, it cures and greatly injures a human body simultaneously. In CPB, hemodilution, the contact between blood and the surface of foreign matter and other various factors activate the stress response in the body and causes tissue edema, increase in total body water and systemic inflammatory response syndrome (SIRS); in severe cases, they may give rise to organ dysfunction. Ultrafiltration (UF) not only can remove the redundant moisture effectively from the body after $\mathrm{CPB}$, concentrate blood cells and recover body fluid equilibrium, but also can clear away part of inflammatory medium, improve postoperative organ function and enhance the clinical effect after an open-heart surgery.
\end{abstract}

Keywords. Cardiopulmonary bypass.

\section{Basic principle of ultrafiltration}

The basic principle of ultrafiltration is to separate and filter out the moisture and soluble small molecular substances in blood from cellular constituents and plasma protein in the blood vessel. The driving force for filtration mainly relies on the transmembrane pressure. Generally, the allowable range of pressure difference on both sides of filter membrane is $100 \sim 500 \mathrm{mmHg}$. The factors influencing the filtration effect are as follows: (1) transmembrane pressure (TMP): according to the Startling Law, the bigger the TMP is, the more liquid it will filter out; that the high limit of TMP is exceeded may lead to red cell rapture and even hemolysis; (2) blood flow volume: the low blood flow will result in that a large number of red cells pile up in hollow fiber, increase the possibility of hemolysis, lead to excessively fast blood flow, and prevent liquid from being filtered out in short time, so it is required to control the flow within $100 \sim 300 \mathrm{ml} / \mathrm{min}$; (3) thickness of membrane; (4) quantity and size of membrane apertures; (5) haematocrit (Hct); (6) temperature.

\section{Filtrate ingredients}

The filtrate, which is equivalent to the original urine of kidney, consists of $\mathrm{K}+, \mathrm{Na}+, \mathrm{Cl}-, \mathrm{UA}$, creatinine, glucose and most inflammatory media. Big molecular substances such as albumin, hemoglobin, fibrinogen and cellular constituent cannot penetrate through the filtering membrane, so they cannot be filtered out.

\section{Types and characteristics of ultrafiltration method}

(C) The Authors, published by EDP Sciences. This is an open access article distributed under the terms of the Creative Commons Attribution License 4.0 (http://creativecommons.org/licenses/by/4.0/). 
3.1 Conventional ultrafiltration (CUF) is the earliest ultrafiltration method. When being used, the ultrafilter is in parallel connection with the $\mathrm{CPB}$; its entry end is connected with the arterial line and is generally connected with the exit on the top of arterial micro sphere filter; its exit end is connected with the venous return passage. Establish the TMP with the vacuum aspiration $(-150 \mathrm{mmHg})$ and control the ultrafiltration flow with the additional pump. The ultrafiltration timing is generally the period from the beginning of rewarming to halt.

Characteristics: It is difficult for normal bypass to maintain the liquid level of oxygenator, so the moisture filtered out is limited; as for a patient with a short duration of operation, it is usually required to terminate the CPB before ultrafiltration.

3.2 Modified ultrafiltration (MUF) is the most common ultrafiltration method, which was created by Naik and other scholars [1] in the 1990s. The entry is connected with the arterial end close to the aortic cannulation with a Y-shaped tee; similarly, the exit end is connected with the venous return line; blood is transfused back to the right atrium; besides, a piezometer tube is equipped at the exit end to monitor the right atrial pressure; a pump is used to control the flow, which is generally within $100 \sim 150 \mathrm{ml} / \mathrm{min}$. The ultrafiltration timing is within $10 \sim 15 \mathrm{~min}$ after the end of CPB.

Characteristics: In case of drop of blood pressure due to undercapacity during ultrafiltration, we can return the residual blood from the oxygenator to the patient through the aortic pump. As the technology is conducted after the CPB stops, the advantages of a certain degree of hemodilution in bypass can be shown; in particular, it is beneficial to patients with high cyanotic blood viscosity; however, once the CPB stops, the low Hct and hemodilution are harmful to infant patients, so it is required to correct them timely; the MUF has such an effect. Free from the influence of the liquid level inside the venous blood storage room and the duration of operation, it can eliminate moisture very thoroughly.

3.3 Zero balance ultrafiltration (ZBUF), which was created by Journois and other scholars [2] in 1996, is a way of ultrafiltration after modifying CUF, so the way to install its filter and the ultrafiltration time are the same with those of CUF. Zero balance, namely, means adding crystalloid solution with the same volume of liquid filtered into the venous blood storage room and filtering out inflammatory media through constant circulation.

Characteristics: It can get rid of inflammatory media effectively and reduce the concentration of inflammatory factors but cannot filter out the unnecessary moisture in vivo to concentrate blood. As shown in Journois's research, the combination of MUF and ZBUF technologies can remove inflammatory media, unnecessary moisture and concentrate blood in a better way. At present, the technology has been applied in many hospitals.

\section{Function of ultrafiltration in CPB}

4.1 Influence of ultrafiltration on water content and HCT in the body: It has been found through the research of Naik [1, 3] that MUF is superior to CUF in water drainage and generally can increase Hct to $40 \%$. Hennein and other scholars [4] studied 38 patients with congenital heart disease in different groups and made comparative observation of CUF as well as the ability of the control group and the group using V-VMUF 3 to drain the body water. The result shows that only MUF can maximally drain body water, raise Hct to a relatively ideal degree and thus shorten patients' average hospitalization period.

4.2 Influence of ultrafiltration on hemorrhage and transfusion: through the large-sample study of Naik [1, 3], it is found that MUF can lower the postoperative blood loss and the dosage of banked blood and plasma; based on analysis, the causes are as follows: (1) hemoconcentration itself can reduce blood loss; (2) the concentration of blood coagulation factors including platelet increases; (3) some thrombolytic substances are filtered out. Friesen and other scholars [5] observed the changes in the coagulation system by conducting MUF for 20 infants whose weight was lower than $15 \mathrm{~kg}$. The result shows that MUF can enhance Hct significantly; both the fibrin and plasma total protein rise relatively significantly; there is no obvious change in platelet; the decrease in postoperative bleeding is because that the application of MUF can alleviate the disorder of children's coagulation system arising from pre-charge. Similarly, Draisma's result also shows that MUF can enhance the Hct level, reduce the dosage of banked blood, and significantly reduce the thoracic drainage fluid of postoperative patient. Koutlas et al [6] made the similar research between January 1995 and June 1996 , showing that there are significant decreases in the dosage of banked blood, pleural effusion, pericardial drainage fluid and 
postoperative mortality of infant patients with MUF. In conclusion, it is certain that MUF can reduce hemorrhage and transfusion in cardiovascular surgery, so it provides us with a way to reduce the use of blood products.

4.3 Influence of ultrafiltration on hemodynamics: MUF, which improves patients' disorder of water metabolism arising from $\mathrm{CPB}$, can help to improve intraoperative and postoperative patients' hemodynamics. Davies et al [7] monitored all preoperative and postoperative ultrasonic indicators of 21 patients through ICE. The result shows that the patients with MUF have their left ventricular function of contraction enhanced after $\mathrm{CPB}$, have their cardiac compliance improved and use less vasoactive drugs than the control group. Chaturvedi [8] made an experimental observation of changes in the left ventricular function by grouping 22 children with congenital heart disease in the CPB process. The result shows that, as for the patients with the application of MUF, their Hct can be increased to $34 \%(21 \% \sim 42 \%)$ and $40 \%$ respectively during and after operation; the proportion curve of end pressure myocardial contraction and capacity (Ees) increases by 58\%; however, there is no significant change in the parameters of the control group. Schlunzen et al [9], Danish scientists, used MUF for $12 \mathrm{~min}$ averagely for 134 infants with the average age of 5 months old, average weight of $5.3 \mathrm{~kg}$ and congenital heart disease; the filtered moisture was $44 \mathrm{ml} / \mathrm{kg}$ on average; the Hct increased from $28 \%$ to $40 \%$; the mean arterial pressure was $56 \sim 74 \mathrm{mmHg}$; the arterial partial pressure of oxygen was $30.8 \sim 34.1 \mathrm{kPa}$; the heart rate decreased from 145 times/min to 136 times/min; however, there's no significant change in their parameters such as CVP and LAP.

4.4 Influence of ultrafiltration on inflammatory medium filtering: During CPB, the contact between blood and the surface of foreign matter results in the occurrence of numerous inflammatory responses, releases abundant inflammatory media, and finally causes injuries to tissue and organ. Ultrafiltration can filter out inflammatory media but the filtering effect of ultrafiltration on inflammatory media is greatly controversial at present.

Naik et al [10] have found through research that ultrafiltration can filter out some toxicants arising from $\mathrm{CPB}$. The capillary electrophoretic analysis on ultrafiltrate shows that there are abundant inflammatory media in the ultrafiltrate, such as all sorts of cell factors, tumor necrosis factors and myocardial necrosis factors. Although Wang [11] and other scholars affirmed the existence of TNF- $\alpha$ and other inflammatory factors in the ultrafiltrate through measurement in their researches, the problem is that the concentration of inflammatory factors in plasma after application of MUF is the same with that before filtration or even increases; it is probably because inflammatory media cannot be filtered out after they are combined with the surface of protein or red cell. Most researches suggest that only ZBUF can filter out inflammatory media effectively. Zhu Deming et al [12] of Shanghai Xinhua Hospital in China compared the application of MUF and ZBUF in infants' CPB. The result shows that all forms of ultrafiltration can filter out inflammatory media but only ZBUF can reduce the plasma concentration of inflammatory media; besides, other forms of ultrafiltration can only achieve hemoconcentration.

\section{Conclusion}

The ultrafiltration technology is a method to effectively concentrate blood and remove excessive moisture and metabolites from the body. The application of ultrafiltration technology during or after CPB can attain a rather satisfactory balance liquid intake and output volume during operation, alleviate the cardiac load, enhance the plasma colloid osmotic pressure, accelerate moisture absorption among tissues, eliminate inflammatory media, alleviate systemic inflammatory response, and play an positive role in preventing clinical or subclinical pulmonary edema, cerebral injury and cardiac insufficiency etc. at the early stage after operation. However, we still cannot completely know the ultrafiltration action mechanism for $\mathrm{CPB}$ cardiac surgery; especially, its influence on cell factors is greatly controversial and further studies are needed. During MUF, the concentration of inflammatory media cannot remain at a rather low level, partly counteracting the effect of ZBUF. It is our new problem to figure out how to use the means of ultrafiltration effectively and how to give play to the ultrafiltration effect on alleviating inflammatory responses in a better way. 


\section{References}

1. Naik SK, Knight A, Elliott MJ.A successful modification of ultrafiltration for cardiopulmonary bypass in children.Perfusion, 1991.6: 41.

2. Journois D, Israel-Biet D, Pouard P, et al.High volume, zero-balanced hemofiltration to reduce delayed inflammatory responce to cardiopulmonary bypass in children.Anesthesiology, 1996.86: 965-976.

3. Naik SK, Elliott MJ.Ultrafiltration and pediatric cardiopulmonary bypass.Perfusion, 1993.8 (1) : 101-112.

4. Hennein HA, Kiziltepe U, Barst S, et al.Venous modified ult-rafiltration after cardiopulmonary bypass in children: a prospective randomized study.J Tborac Cardixasc Surg, 1999.117: 496-505.

5. Friesen RH, Campbell DN, Clarke DR, et al.Modified ultrafiltration attenuates dilutional coagulopathy in pediatric open heart operations.Ann Thorac Surg, 1997.64: 1787.

6. Koutlas TC, Gavnor JW, Nicolson SC, et al.Modified ultrafiltration reduces postoperative morbidity aftet cavopulmonary connection.Ann Thorac Surg, 1997.64 (1) : 37-42.

7. Davies MJ, Nguyen K, Gaynor JW, et al.Modified ultrafiltration improves left ventricular systolic function in infants after cardiopulmonary bypass.J Thorac Cardiovasc Surg, 1998.115 (2) : 361-369.

8. Chaturvedi RR, Shore DF, White PA, et al.Modified ultrafiltration improves global left ventricular systolic function after open-heart surgery in infants and children.Eur J Cardiothorac Surg, 1999.15（6）: 742-746.

9. Schlunzen L, Pedersen J, Hjortholm K, et al.Modified ultrafiltration in paediatric cardiac surgery.Perfusion, 1998.13 (2) : 105-109.

10. Naik SK, Knight A, Elliott MJ.A prospective randomized study of a modified technique of ultrafiltration during pediatric open-heart surgery.Circulation, 1999.84 (suppl 3) : 422.

11. Wang MJ, Chiu IS, Hsu CM, et al.Efficacy of ultrafiltration in removing inflammatory mediator during pediatric cardiac operations.Ann Thorac Surg, 1996.61: 651-666.

12. Ming ZD, Wei W, Hong C, et al.Blanced ultrafiltration, modified ultrafiltration, and balanced ultrafiltration with modified ultrafiltration in pediatric cardiopulmonary bypass.J Extra Corpor Technol, 2001.33 (4) : 223. 